\title{
A Discrete Model for Correlation Between Quantization Noises
}

\author{
Jean-Charles Naud, Student Member, IEEE, Daniel Ménard, Member, IEEE, \\ Gabriel Caffarena, Senior Member, IEEE, and Olivier Sentieys, Member, IEEE
}

\begin{abstract}
The automation of fixed-point conversion requires fast methods to evaluate the numerical accuracy of the system. As an alternative to a simulation-based approach, most of the analytical methods use perturbation theory to provide the expression of the quantization noise at the output of a system. Most existing analytical methods do not consider a correlation between noise sources. This assumption is no longer valid when a unique datum is quantized several times. This brief proposes to study the correlation between quantization noises with different quantization modes (truncation and rounding) and considering the number of eliminated bits. Then, the expression of the power of the output quantization noise is provided when the correlation between the noise sources is considered. The proposed approach allows improving significantly the estimation of the output quantization noise power compared to the classical approach, with a slight increase of the computation time. In our experiment, the maximal relative estimation error obtained with the proposed approach is less than $2 \%$ compared to $84 \%$ when a correlation is not taken into account.
\end{abstract}

Index Terms-Correlation, fixed-point arithmetic, numerical accuracy evaluation, quantization noise, round-off noise.

\section{INTRODUCTION}

$\mathbf{F}$ IXED-POINT arithmetic is widely used in embedded systems to reduce implementation costs like execution time, area, and power consumption. Fixed-point conversion is composed of two main steps corresponding to dynamic range evaluation and word-length (WL) optimization. The aim of WL optimization is to minimize the implementation cost as long as the effects of finite precision are acceptable. This optimization process is based on an iterative procedure where the numerical accuracy is evaluated a great number of times. Thus, efficient methods are required to evaluate this numerical accuracy to limit the optimization time.

To evaluate numerical accuracy, approaches based on fixedpoint simulations are generic, but they also lead to long execution time. Thus, the search space is drastically limited, and suboptimal solutions are obtained. Analytic methods reduce

Manuscript received November 9, 2011; revised February 10, 2012; accepted October 1, 2012. Date of publication November 16, 2012; date of current version January 4, 2013. This work was supported by the Nano 2012 $\mathrm{R} \& \mathrm{D}$ research program in collaboration with STmicroelectronics. This brief was recommended by Associate Editor O. Gustafsson.

J.-C. Naud, D. Ménard, and O. Sentieys are with Inria, Irisa, University of Rennes, 22305 Lannion, France (e-mail: jean-charles.naud@irisa.fr; daniel.menard@irisa.fr; olivier.sentieys@irisa.fr).

G. Caffarena is with Universidad CEU San Pablo, 28668 Boadilla del Monte, Spain (e-mail: gabriel.caffarenafernandez@ceu.es).

Color versions of one or more of the figures in this brief are available online at http://ieeexplore.ieee.org.

Digital Object Identifier 10.1109/TCSII.2012.2222838 significantly the evaluation time by providing the mathematical expression of a metric equivalent to the numerical accuracy. The output quantization noise power is widely used as a relevant metric for evaluating the numerical accuracy.

Most existing methods do not take into account the correlation between the different quantization noise sources (QNSs), which is due to multiple quantizations of the same data. Consequently, the quality of the estimation can be degraded when multiple quantizations occur and the solution obtained from the WL optimization process can lead to an implementation cost overhead. In [1], graph transformations are applied to handle the correlation. This technique can become complex and is valid only for truncation. In [2], the expression of the correlation is provided but for a very specific case.

In this brief, the expressions of the correlation and the covariance, considering the number of eliminated bits, are proposed for truncation and rounding. Then, to take correlation into account, the expression of the output quantization noise power is provided. This model extends existing methods by integrating the covariance between the noise sources and leads to a slight increase in complexity.

This brief is organized as follows. Existing works handling correlation between QNSs are presented in Section II. In Section III, quantization modes corresponding to truncation and rounding are defined. The expressions of the correlation and the covariance between QNSs are detailed in Section IV. The expression of the global output quantization noise considering the correlation is provided in Section V. The quality of the proposed models is analyzed through experiments in Section VI, while Section VII concludes this brief.

\section{RELATED WORK}

Correlation between QNSs occurs when a datum $x_{0}$ is quantized several times. Fig. 1 shows such an example where $x_{i}$ is the datum after each quantization $\mathcal{Q}_{i}$ with $i$ equal to one or two. $\mathcal{Q}_{i}$ leads to an unavoidable quantization error $e_{i}$ between the values of the data $x_{i}$ and $x_{0} . e_{i}$ can be assimilated to a noise source, and we denote $E_{i}$ as the random variable corresponding to this error. Let $w_{i}$ denote the fractional part $\mathrm{WL}$ of the datum $x_{i}$ and $q_{i}$ denote the quantization step associated to $x_{i}$. $q_{i}=2^{-w_{i}}$ with $w_{i}$ as the weight of the least significant bit. The number of bits eliminated during the quantization process $\mathcal{Q}_{i}$ is defined as $k_{i}$. The relation between the quantization steps $q_{i}$ and $q_{0}$ is $q_{i}=2^{k_{i}} q_{0}$, with $i$ equal to one or two. If $x_{0}$ is in infinite precision, $q_{0}$ is equal to zero and $k_{1}$ and $k_{2}$ tend to infinity. Let $X_{i}$ denote the set containing all the values that can be represented in the fixed-point format after the quantization $\mathcal{Q}_{i}$. 


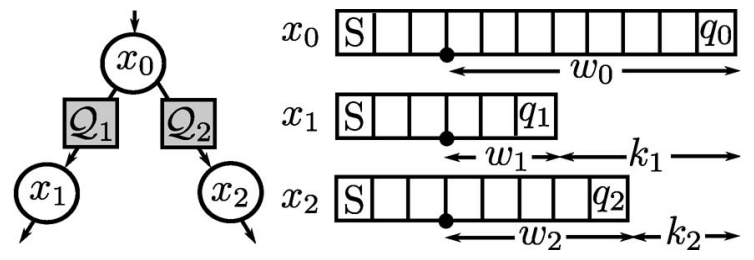

Fig. 1. Quantized data representation.

Given that $k_{2}$ bits are common between the QNSs $e_{1}$ and $e_{2}$, these QNSs are correlated. Let $y$ denote the output of the global targeted system. Let $H_{i}$ denote the system having $e_{i}$ as input and $y$ as output. As the QNS $e_{i}$ propagates through the system $H_{i}$, correlation between different QNSs $e_{i}$ obviously influences the output noise power and has, therefore, to be considered for a precise numerical accuracy evaluation.

In [1], the problem of correlation between different QNSs is handled through signal flow graph transformations. If $k_{1}>k_{2}$, the quantization processes $\mathcal{Q}_{1}$ and $\mathcal{Q}_{2}$ are transformed into two quantization processes $\mathcal{Q}_{1}^{\prime}$ and $\mathcal{Q}_{0}^{\prime}$. The quantization process $\mathcal{Q}_{0}^{\prime}$, corresponding to the elimination of $k_{2}$ bits, is inserted just after the datum $x_{0}$. The associated noise source $e_{0}^{\prime}$ propagates through the system corresponding to the combination of the systems $H_{1}$ and $H_{2}$. The modified quantization process $\mathcal{Q}_{1}^{\prime}$ corresponds to the elimination of $k_{1}-k_{2}$ bits. The associated noise source $e_{1}^{\prime}$ propagates through the system $H_{1}$. As the quantization process $\mathcal{Q}_{2}$ disappears, the noise sources $e_{1}^{\prime}$ and $e_{0}^{\prime}$ are considered not correlated, and classical techniques can be used to estimate the output quantization noise. First, this technique is only valid for truncation. Indeed, a quantization with rounding cannot be decomposed into two independent quantization processes. Second, the system considered for noise propagation depends on the values of $k_{2}$ and $k_{1}$. Therefore, to have a generic model, all the combinations between the systems $H_{i}$ have to be considered. If one unique datum is quantized $P$ times, the number of systems to consider is $P !+1$, so growing up exponentially and making more complex the expression of the output quantization noise.

In [2], the correlation between two quantization noises of one datum after multiplication by constants is studied. The correlation between the quantization noise after the multiplication of $x_{1}$ by a constant $A_{1}$ and the quantization noise after the multiplication of $x_{2}$ by constant $A_{2}$ is analyzed. Only the case of rounding and the case of linear time-invariant systems are considered. Moreover, the two quantization steps are identical, and only $A_{1}$ and $A_{2}$ can be different.

In this brief, a correlation model considering different quantization modes (truncation and rounding), different quantization steps, and different kinds of input $\left(x_{0}\right.$ is in infinite or finite precision) is proposed. The proposed approach modifies the expression of the output quantization noise by adding one more term but with a slight increase in the complexity to calculate this expression.

\section{Quantization Mode Description}

In this section, the probability density function (pdf) and the statistical moments of the QNSs generated during a quantization process are presented for the truncation and rounding quantization modes in the case of two's complement coding. The
TABLE I

MEAN AND VARIANCE FOR THE TwO QuANTIZATION MOdES

\begin{tabular}{c|c|c}
\hline \hline Quantization mode $Q_{1}$ & Mean & Variance \\
\hline Truncation & $\frac{q_{1}}{2} \cdot\left(1-2^{-k_{1}}\right)$ & $\frac{q_{1}^{2}}{12} \cdot\left(1-2^{-2 k_{1}}\right)$ \\
Rounding & $-\frac{q_{1}}{2} \cdot\left(2^{-k_{1}}\right)$ & $\frac{q_{1}^{2}}{12} \cdot\left(1-2^{-2 k_{1}}\right)$ \\
\hline \hline
\end{tabular}

quantization process $\mathcal{Q}_{1}$, shown in Fig. 1, is under consideration. The quantization error $e_{1}$ resulting from the quantization process $\mathcal{Q}_{1}$ is defined as

$$
e_{1}=x_{0}-x_{1}
$$

By using the model by Widrow [3], [4], $e_{1}$ can be assimilated to an additive white noise, uniformly distributed, which is uncorrelated to the signal.

1) Truncation: In the case of truncation, the datum $x_{0}$ is always rounded toward the lower value available in the set $X_{1}$ and becomes

$$
x_{1}=\left\lfloor x_{0} \cdot q_{1}^{-1}\right\rfloor \cdot q_{1}=t \cdot q_{1} \forall x_{0} \in\left[t \cdot q_{1},(t+1) \cdot q_{1}[\right.
$$

with $\lfloor\cdot\rfloor$ as the floor function defined as $\left\lfloor x_{0}\right\rfloor=\max (n \in$ $\left.\mathbb{Z} \mid n \leq x_{0}\right)$ and with $q_{1}$ as the quantization step. The pdf of the QNS $p_{E_{1}}\left(e_{1}\right)$ is given by (3) with $\delta$ as the Kronecker delta

$$
p_{E_{1}}\left(e_{1}\right)=\frac{1}{2^{k_{1}}} \sum_{j=0}^{2^{k_{1}}-1} \delta\left(e_{1}-j \cdot q_{0}\right) .
$$

2) Rounding: Rounding the quantization mode rounds the value $x_{0}$ to the nearest value available in the set $X_{1}$ as

$$
x_{1}=\left\lfloor\left(x_{0}+\frac{1}{2} q_{1}\right) \cdot q_{1}^{-1}\right\rfloor \cdot q_{1} .
$$

The midpoint $q_{m}=(t+(1 / 2)) \cdot q_{1}$ between $t \cdot q_{1}$ and $(t+$ $1) \cdot q_{1}$ is always rounded up to the higher value $(t+1) \cdot q_{1}$. For this quantization mode, the $\operatorname{pdf} p_{E_{1}}\left(e_{1}\right)$ is given by

$$
p_{E_{1}}\left(e_{1}\right)=\frac{1}{2^{k_{1}}} \sum_{j=-2^{k_{1}-1}}^{2^{k_{1}-1}-1} \delta\left(e_{1}-j \cdot q_{0}\right) \text {. }
$$

From [5] and [6], mean and variance expressions are given in Table I for each quantization mode $Q_{1}$. If $x_{0}$ has a continuous amplitude, as in analog-to-digital conversion, $k_{1}$ is considered as $+\infty$.

\section{Correlation And Covariance Expressions}

In this section, the expressions of the correlation and the covariance between two QNSs $e_{1}$ and $e_{2}$, resulting from the quantization of one unique datum $x_{0}$ as shown in Fig. 1, are determined. The covariance is used in the expression of the global output quantization noise power to improve the quality of the noise power estimation. When the datum $x_{0}$ is quantized $P$ times, $P(P-1) / 2$ correlations, between the $P$ QNSs, have to be studied. The reasoning to determine the correlation and covariance expressions is detailed in the following with $k_{1} \geq$ $k_{2}$ and for the cases where $\mathcal{Q}_{1}$ is a truncation $(\mathrm{T})$ and $\mathcal{Q}_{2}$ is a rounding (R). The two discrete pdfs $p_{E_{1}}$ and $p_{E_{2}}$ of the QNSs 


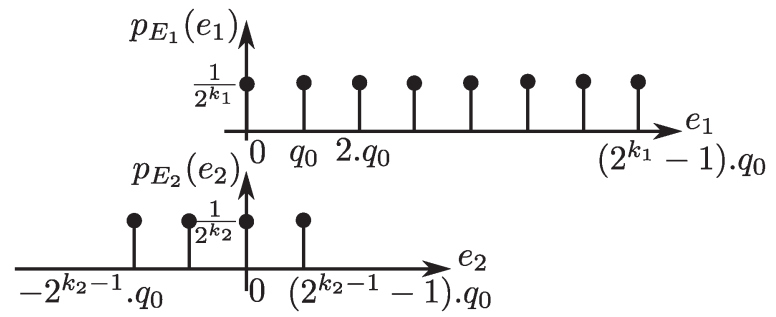

Fig. 2. A pdf of the QNSs $e_{1}$ and $e_{2}$ for $\mathcal{Q}_{1}=T, \mathcal{Q}_{2}=R, k_{1}=3$, and $k_{2}=2 . q_{0}$ is the quantization step.

$e_{1}$ and $e_{2}$, respectively, are shown in Fig. 2 for the cases of $k_{1}=3$ and $k_{2}=2$.

Let $E_{1}$ and $E_{2}$ denote the discrete random variables corresponding to the QNSs. The correlation $E\left[E_{1} \cdot E_{2}\right]$ is determined from $p_{E_{1}, E_{2}}$ the joint pdf between $E_{1}$ and $E_{2}$ as

$$
E\left[E_{1} \cdot E_{2}\right]=\sum_{i} \sum_{j} i \cdot q_{0} \cdot j \cdot q_{0} \cdot p_{E_{1}, E_{2}}\left(e_{1}=i \cdot q_{0}, e_{2}=j \cdot q_{0}\right)
$$

where $i$ and $j$ enumerate the possible events of $e_{1}$ and $e_{2}$.

The joint pdf $p_{E_{1}, E_{2}}$ is obtained from $p_{E_{1} \mid E_{2}}$ which is the conditional probability of $E_{1}$ given $E_{2}$ as

$$
p_{E_{1}, E_{2}}\left(e_{1}, e_{2}\right)=p_{E_{1} \mid E_{2}}\left(e_{1} \mid e_{2}\right) \cdot p_{E_{2}}\left(e_{2}\right) .
$$

For each value of $e_{2}, 2^{k_{1}-k_{2}}$ values are obtained for $e_{1}$; thus

$$
\begin{aligned}
& p_{E_{1} \mid E_{2}}= \frac{1}{2^{k_{1}-k_{2}}} \cdot \\
& \times\left[\sum_{j=0}^{2^{k_{2}-1}-1} \delta\left(e_{2}-j q_{0}\right) \sum_{t=0}^{2^{k_{1}-k_{2}-1}} \delta\left(e_{1}-e_{2}-t \cdot q_{2}\right)\right. \\
& \quad+\sum_{j=-2^{k_{2}-1}}^{-1} \delta\left(e_{2}-j q_{0}\right) \\
&\left.\quad \times \sum_{t=0}^{2^{k_{1}-k_{2}-1}} \delta\left(e_{1}-e_{2}-(t+1) \cdot q_{2}\right)\right]
\end{aligned}
$$

From (7) and (6), the correlation between $E_{1}$ and $E_{2}$ becomes

$$
\begin{aligned}
E\left[E_{1} \cdot E_{2}\right]= & \frac{q_{0}^{2}}{2^{k_{1}}} \sum_{t=0}^{2^{k_{1}-k_{2}-1}} \sum_{j=0}^{2^{k_{2}-1}-1} j\left(j+t \cdot 2^{k_{2}}\right) \\
& +\left(j-2^{k_{2}-1}\right)\left(j+2^{k_{2}-1}+t \cdot 2^{k_{2}}\right) \\
= & -\frac{q_{2}^{2}}{24}+\frac{q_{0}^{2}}{6}-\frac{q_{0} \cdot q_{1}}{4} .
\end{aligned}
$$

The covariance $\operatorname{cov}\left(E_{1}, E_{2}\right)$ is determined from the correlation term $E\left[E_{1} \cdot E_{2}\right]$ as

$$
\operatorname{cov}\left(E_{1}, E_{2}\right)=E\left[E_{1} \cdot E_{2}\right]-E\left[E_{1}\right] \cdot E\left[E_{2}\right] .
$$

The term $E\left[E_{1}\right] \cdot E\left[E_{2}\right]$ can be computed from the equations given in Table I and is equal to

$$
\begin{aligned}
E\left[E_{1}\right] \cdot E\left[E_{2}\right] & =\frac{q_{1}}{2}\left(1-2^{-k_{1}}\right) \frac{q_{2}}{2}\left(-2^{-k_{2}}\right) \\
& =\frac{q_{0}^{2}-q_{0} \cdot q_{1}}{4} .
\end{aligned}
$$

TABLE II

CORRELATION AND COVARIANCE EXPRESSIONS FOR THE DIFFERENT QUANTIZATION MODES OF (T) TRUNCATION OR (R) ROUNDING AND FOR DIFFERENT CONDITIONS ON $k_{1}$ AND $k_{2}$

\begin{tabular}{c|c|c||c|c}
\hline \hline$Q_{1}$ & $Q_{2}$ & Condition & $\mathrm{E}\left[E_{1} \cdot E_{2}\right]$ & $\operatorname{cov}\left(E_{1}, E_{2}\right)$ \\
\hline $\mathrm{T}$ & $\mathrm{T}$ & $k_{1} \geq k_{2}$ & $\frac{q_{2}^{2}}{12}+\frac{q_{0}^{2}}{6}-\frac{q_{0} \cdot q_{2}}{4}$ & $\frac{q_{2}^{2}}{12}-\frac{q_{0}^{2}}{12}$ \\
& & & $-\frac{q_{0} \cdot q_{1}}{4}+\frac{q_{1} \cdot q_{2}}{4}$ & $-\frac{q_{2}^{2}}{24}+\frac{q_{0}^{2}}{6}-\frac{q_{0} \cdot q_{1}}{4}$ \\
$\mathrm{~T}$ & $\mathrm{R}$ & $k_{1} \geq k_{2}$ & $-\frac{q_{2}^{2}}{24}-\frac{q_{0}^{2}}{12}$ \\
$\mathrm{R}$ & $\mathrm{T}$ & $k_{1}>k_{2}$ & $\frac{q_{2}^{2}}{12}+\frac{q_{0}^{2}}{6}-\frac{q_{0} \cdot q_{2}}{4}$ & $\frac{q_{2}^{2}}{12}-\frac{q_{0}^{2}}{12}$ \\
$\mathrm{R}$ & $\mathrm{R}$ & $k_{1}=k_{2}$ & $\frac{q_{2}^{2}}{12}+\frac{q_{0}^{2}}{6}$ & $\frac{q_{2}^{2}}{12}-\frac{q_{0}^{2}}{12}$ \\
$\mathrm{R}$ & $\mathrm{R}$ & $k_{1}>k_{2}$ & $-\frac{q_{2}^{2}}{24}+\frac{q_{0}^{2}}{6}$ & $-\frac{q_{2}^{2}}{24}-\frac{q_{0}^{2}}{12}$ \\
\hline \hline
\end{tabular}

Thus, from (9) and (11), the expression of the covariance becomes

$$
\operatorname{cov}\left(E_{1}, E_{2}\right)=-\frac{q_{2}^{2}}{24}-\frac{q_{0}^{2}}{12}
$$

Given that $k_{1} \geq k_{2}$, the term $q_{1}$ is eliminated because just the $k_{2}$ least significant bits are common between $e_{1}$ and $e_{2}$. The correlation and covariance expressions are given in Table II for the different quantization modes $Q_{i}$ corresponding to truncation $(\mathrm{T})$ or rounding $(\mathrm{R})$ and for different conditions on $k_{1}$ and $k_{2}$. If $x_{0}$ is in infinite precision, $q_{0}$ is equal to zero.

\section{OutPut Quantization Noise Power}

Different models have been proposed to estimate the power of the quantization noise at the output of a system [7]-[10]. These approaches do not consider the correlation between quantization noises, but they can be easily extended to integrate this correlation to improve the estimation quality.

From [8], the output quantization noise $e_{y}$ is the sum of the contributions of the $N$ QNSs $e_{i}$

$$
e_{y}(n)=\sum_{i=1}^{N} \sum_{t=0}^{\infty} h_{i}(t, n) \cdot e_{i}(n-t)
$$

where $h_{i}$ corresponds to the time-varying impulse response of the system $H_{i}$ between $e_{i}$ and the output $y$.

The power $P_{e_{y}}$ of the output quantization noise is obtained by determining the second-order moment of $e_{y}$ with a similar derivation as in [8]. From (13), the expression of $P_{e_{y}}$ is

$$
\begin{aligned}
P_{e_{y}}= & E\left[E_{y}^{2}\right] \\
= & \sum_{i=1}^{N} K_{i} \cdot \sigma_{i}^{2}+\sum_{i=1}^{N} \sum_{j=1}^{N} L_{i j} \cdot \mu_{i} \cdot \mu_{j} \\
& +\sum_{i=1}^{N} \sum_{\substack{j=1 \\
j \neq i}}^{N} M_{i j} \cdot \operatorname{cov}\left(E_{i}, E_{j}\right)
\end{aligned}
$$

with

$$
K_{i}=\sum_{t=0}^{\infty} E\left[h_{i}^{2}(t, n)\right]
$$




$$
\begin{aligned}
L_{i j} & =\sum_{t=0}^{\infty} \sum_{v=0}^{\infty} E\left[h_{i}(t, n) h_{j}(v, n)\right] \\
M_{i j} & =\sum_{t=0}^{\infty} E\left[h_{i}(t, n) h_{j}(t, n)\right]
\end{aligned}
$$

and where $K_{i}, L_{i j}$, and $M_{i j}$ are constant terms depending only on the system in infinite precision, which can thus be determined only once. The variance $\sigma_{i}^{2}$, the mean $\mu_{i}$, and the covariance $\operatorname{cov}\left(E_{i}, E_{j}\right)$ between QNSs depend on the quantization modes, the number of bit $w_{i}$ for the fractional part, and the number of bits eliminated $k_{i}$ for each datum $x_{i}$. The quantization of a unique datum $P$ times leads to $P$ QNSs and to $P(P-1)$ nonnull terms $M_{i j}$.

Compared to existing methods, a new term $M_{i j} \cdot \operatorname{cov}\left(E_{i}, E_{j}\right)$ is introduced. The covariance $\operatorname{cov}\left(E_{i}, E_{j}\right)$ is not equal to zero when both quantization processes $\mathcal{Q}_{i}$ and $\mathcal{Q}_{j}$ quantize the same data and when the delay inside the systems $H_{i}$ and $H_{j}$ is the same because the QNSs are white. Otherwise, $\operatorname{cov}\left(E_{i}, E_{j}\right)$ is equal to zero. The terms $M_{i j}$ are not computed when $\operatorname{cov}\left(E_{i}, E_{j}\right)$ is always equal to zero. Thus, the increase in time due to the computation of $M_{i j}$ depends on the number of QNSs, which can be correlated. As $M_{i j} \cdot \operatorname{cov}\left(E_{i}, E_{j}\right)$ can be positive or negative, if the quantization correlation is not considered, the quantization noise power can be overestimated or underestimated.

This model is valid for systems made up of smooth operations and if the input signals satisfy the conditions associated to the model by Widrow and Kollár [3]. An operation is smooth if its associated function is differentiable.

\section{EXPERIMENTS}

In this section, the estimations obtained with the proposed models are compared with the results obtained by the equivalent simulations, which are considered as the reference. To obtain relevant statistics, $10^{7}$ samples are used to simulate the systems. The system is simulated in fixed point and in double-precision floating point. The error $e_{i}$ associated with a datum $x_{i}$ is obtained by subtracting the floating-point values and the fixedpoint values. The floating-point simulation is considered as the reference as it is a reliable approximation of the infinite precision. Indeed, the error due to floating-point arithmetic can be neglected compared to the error due to the fixed point when the data WLs are small.

\section{A. Correlation Between QNSs}

In this section, the quality of the proposed model to estimate the covariance given in (10) and in Table II is evaluated. For the sake of clarity, $\rho$, the correlation coefficient between the QNSs $e_{1}$ and $e_{2}$ resulting from the quantization of $x_{0}$ as in Fig. 1, is analyzed. $\rho$ is determined from the covariance $\operatorname{cov}\left(E_{1}, E_{2}\right)$ and the standard deviations $\sigma_{1}$ and $\sigma_{2}$ of noises $e_{1}$ and $e_{2}$, and it is defined as

$$
\rho=\left\{\begin{array}{cl}
\frac{\operatorname{cov}\left(E_{1}, E_{2}\right)}{\sigma_{1} \cdot \sigma_{2}}, & \text { if } \sigma_{1} \neq 0 \text { and } \sigma_{2} \neq 0 \\
0, & \text { else. }
\end{array}\right.
$$

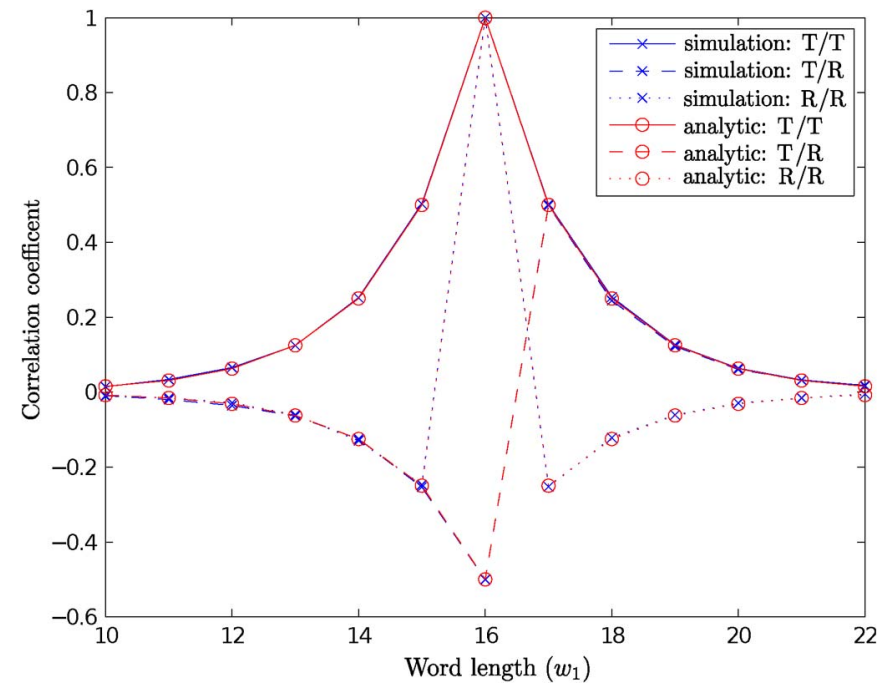

Fig. 3. Correlation coefficient $\rho$ obtained with the proposed model and by simulations for different quantization modes and for different values of the fractional part WLs $w_{1}$.

The correlation coefficient $\rho$ is evaluated for three combinations of rounding modes and for different fractional part WLs $w_{1}$ and $w_{2}$ of the data $x_{1}$ and $x_{2}$, respectively. $w_{2}$ is set to 16 bits, $w_{1}$ varies from 10 to 22 bits, and $x_{0}$ is considered as a continuous amplitude datum (i.e., $w_{0}=+\infty$ ). In the first case, $Q_{1}$ and $Q_{2}$ are both truncations (T/T). In the second case, $Q_{1}$ is a truncation, and $Q_{2}$ is a rounding (T/R). In the third case, $Q_{1}$ and $Q_{2}$ are rounding $(\mathrm{R} / \mathrm{R})$.

The values of the correlation coefficients obtained with the proposed model and by simulations are shown in Fig. 3 for different values of the fractional part WLs $w_{1}$. The results show that the difference between the proposed model and the reference is very small. The maximal relative error of the proposed estimation is below $1.5 \%$.

The amplitude of the correlation coefficient is maximal when the two WLs $w_{1}$ and $w_{2}$ are equal. In this case, $|\rho|$ is equal to one when the two quantization modes are identical, and $|\rho|$ is equal to $1 / 2$ when the two quantization modes are different. For $Q_{1}=T$ and $Q_{2}=R,|\rho|$ is equal to $1 / 2$ when $w_{1}=w_{2}$ or $w_{1}=w_{2}-1$. The correlation coefficients reduce when the difference between the WLs $w_{1}$ and $w_{2}$ increases.

\section{B. Estimation of the Output Quantization Noise Power}

In this section, the quality of the proposed approach to estimate the output quantization noise with (14) is evaluated and compared with the quality of a classical approach, which does not consider the correlation [in this case, $\operatorname{cov}\left(E_{i}, E_{j}\right)=$ $0 \forall i, j$ in (14)]. To illustrate the benefit of the proposed approach to improve the estimation quality of $P_{e_{y}}$ compared to a classical approach, the computation of a third-order polynomial is considered. This polynomial is $p(x)=0.99998-0.99765$. $x+0.949615 \cdot x^{2}-0.63603 \cdot x^{3}$. The expression of the output quantization noise can be found in [11]. For data having multiple quantizations, half of the quantization is carried out with a WL set to 30 bits and the remaining quantizations are carried out with a WL $w_{T}$ varying from 25 to 35 bits. To analyze 


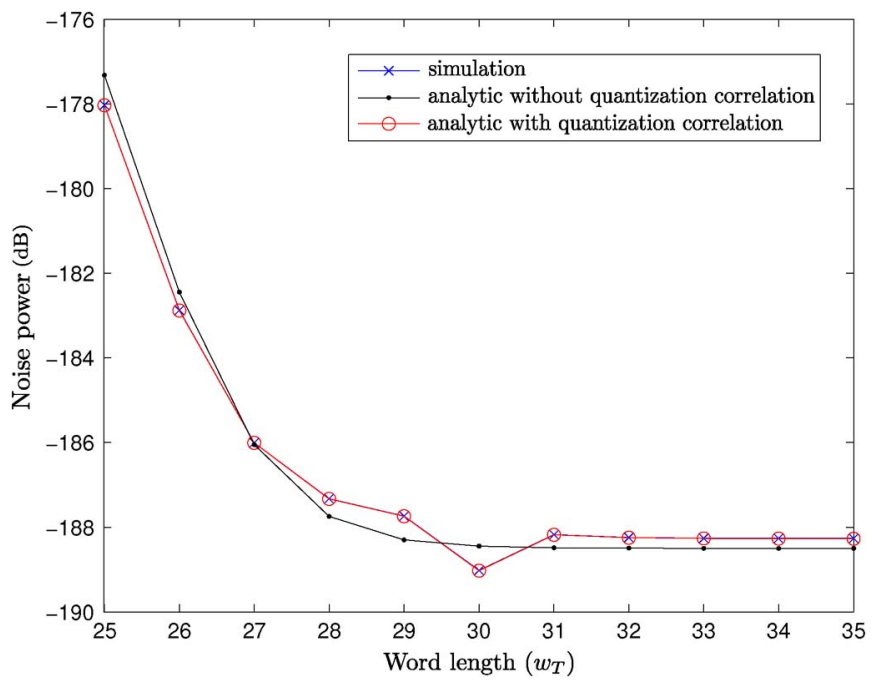

Fig. 4. Accuracy degradation for the polynomial $p(x)$ computation.

correlation effects, the WL of the other data is set such as no bit is eliminated. Quantization modes are selected randomly.

The real values of $P_{e_{y}}$, obtained by simulation, and the estimation of $P_{e_{y}}$, obtained with the proposed approach and with a classical approach, are shown in Fig. 4 for different WLs $w_{T}$. The results show that the estimations obtained with the proposed approach and the reference are very close. In this example, the maximal value of the relative estimation error (REE) is lower than $1 \%$ with the proposed approach and can reach up to $12 \%$ for a classical approach.

The REE is evaluated on different benchmarks. Let $R_{w}$ denote the REE of the proposed approach and $R_{w o}$ denote the REE of a classical approach. For the different benchmarks, a digital signal processor-like architecture is considered. The WL of the multiplication input is set to 16 bits. The WL of the multiplication output and the addition input and output is set to 32 bits. To evaluate $R_{w}$ and $R_{w o}, N_{c q}$ different combinations of quantization modes are tested. The number of correlated QNSs $N_{c}$ is given and can be compared to $N$, the number of QNSs in the application. The execution time to obtain the expression of the output quantization noise power is measured for the proposed approach and for the classical approach. Let $I_{w / w o}$ denote the increase in time of the proposed approach compared to the classical approach. The mean and maximal values of $R_{w}$ and $R_{w o}$ are presented in Table III for six applications, which are a third-order polynomial computation with the direct form (Poly. 3 direct) and the Horner scheme (Poly. 3 Horner), a fifthorder polynomial computation with Horner scheme (Poly. 5 Horner), an $L 2$-norm of an $l$-length vector $(l=4)$, a secondorder Voltera filter, and a function approximation. The function $1 /(1+x)$ is approximated in the interval $[0,1]$ with four thirdorder polynomials as described in [12].

Compared to the classical approach, the proposed estimation leads to an accurate estimation. The maximal value of $R_{w}$ is $1.9 \%$ instead of $84 \%$ for $R_{w o}$. For the polynomial computation, when the correlation is not taken into account, the overestimation leads to an energy consumption overhead between $10 \%$ and $18 \%$ depending on the accuracy constraint. As shown by the results, the increase in time is limited and is less than $8.9 \%$ for the different tested applications. Indeed, the number of terms
TABLE III

REE FOR THE OUTPUT QUANTIZATION

\begin{tabular}{l|lll|ll|ll|l}
\hline \hline & \multirow{2}{*}{$N_{c q}$} & $N$ & $N_{c}$ & \multicolumn{2}{|c|}{$R_{w o}$} & \multicolumn{2}{|c|}{$R_{w}$} & \multirow{2}{*}{ mean } \\
& & & & $\max$ & mean & $\max$ & $I_{w / w o}$ \\
\hline Poly. 3 direct & 64 & 14 & 6 & $9.3 \%$ & $40.1 \%$ & $0.4 \%$ & $1.3 \%$ & $+6.4 \%$ \\
Poly. 3 Horner & 8 & 9 & 3 & $12.7 \%$ & $30.6 \%$ & $0.1 \%$ & $0.2 \%$ & $+7.4 \%$ \\
Poly. 5 Horner & 32 & 15 & 5 & $18.1 \%$ & $79.9 \%$ & $0.1 \%$ & $1.4 \%$ & $+8.9 \%$ \\
Function approx. & 256 & 56 & 24 & $22.4 \%$ & $84.1 \%$ & $1.3 \%$ & $1.9 \%$ & $+1.3 \%$ \\
Vector L2-norm & 256 & 15 & 8 & $7.1 \%$ & $49.8 \%$ & $0.23 \%$ & $0.9 \%$ & $+3.2 \%$ \\
Volterra filter & 256 & 42 & 20 & $14.3 \%$ & $63.7 \%$ & $0.3 \%$ & $1.4 \%$ & $+0.7 \%$ \\
\hline \hline
\end{tabular}

$M_{i j}$ to compute depends on $N_{c}$ and not on $N$. Moreover, each $M_{i j}$ term is less complex than the term $L_{i j}$. The proposed approach allows improving significantly the estimation of the accuracy $\left(P_{e_{y}}\right)$ compared to the classical approach, with a negligible increase in the computation time.

\section{CONCLUSION}

In the context of numerical accuracy evaluation of fixed-point systems, the expressions of the correlation and the covariance between QNSs resulting from the quantization of one unique datum have been proposed in this brief. The model considers the number of eliminated bits and the quantization mode. A maximal relative error of the proposed estimation below $2 \%$ is reported. This model extends existing analytical methods with a negligible increase in the complexity of the evaluation. The expression of the global output quantization noise integrates correlation between QNSs, which improves the quality of the estimation of the output quantization noise compared to existing approaches.

\section{REFERENCES}

[1] G. Constantinides, P. Cheung, and W. Luk, "Wordlength optimization for linear digital signal processing," IEEE Trans. Comput.-Aided Design Integr. Circuits Syst., vol. 22, no. 10, pp. 1432-1442, Oct. 2003.

[2] S. Parker and P. Girard, "Correlated noise due to roundoff in fixed point digital filters," IEEE Trans. Circuits Syst., vol. CAS-23, no. 4, pp. 204211, Apr. 1976.

[3] B. Widrow and I. Kollár, Quantization Noise: Roundoff Error in Digital Computation, Signal Processing, Control, and Communications. Cambridge, U.K.: Cambridge Univ. Press, 2008.

[4] A. Sripad and D. L. Snyder, "A necessary and sufficient condition for quantization error to be uniform and white," IEEE Trans. Acoust., Speech, Signal Process., vol. 25, no. 5, pp. 442-448, Oct. 1977.

[5] G. Constantinides, P. Cheung, and W. Luk, "Truncation noise in fixedpoint SFGs," Electron. Lett., vol. 35, no. 23, pp. 2012-2014, Nov. 1999.

[6] D. Menard, D. Novo, R. Rocher, F. Catthoor, and O. Sentieys, "Quantization mode opportunities in fixed-point system design," in Proc. EUSIPCO, Aalborg, Denmark, Aug. 2010, pp. 542-546.

[7] C. Shi and R. Brodersen, "A perturbation theory on statistical quantization effects in fixed-point DSP with non-stationary inputs," in Proc. IEEE ISCAS, Vancouver, BC, Canada, May 2004, pp. 373-376.

[8] R. Rocher, D. Menard, P. Scalart, and O. Sentieys, "Analytical accuracy evaluation of fixed-point systems," in Proc. EUSIPCO, Poznan, Poland, Sep. 2007, pp. 999-1003.

[9] P. Fiore, "Efficient approximate wordlength optimization," IEEE Trans. Comput., vol. 57, no. 11, pp. 1561-1570, Nov. 2008.

[10] G. Caffarena, J. López, A. Fernandez, and C. Carreras, "SQNR estimation of fixed-point DSP algorithms," EURASIP J. Adv. Signal Process., vol. 2010, pp. 1-13, Feb. 2010.

[11] J. Naud, "Numerical accuracy evaluation for polynomial computation," INIRA, Tech. Rep. 7878, Feb. 2012.

[12] N. Brisebarre, J.-M. Muller, and A. Tisserand, "Computing machineefficient polynomial approximations," ACM Trans. Math. Softw., vol. 32, no. 2, pp. 236-256, Jun. 2006. 